\title{
Repurposing auranofin to treat TP53-mutated or PTEN-deleted refractory B-cell lymphoma
}

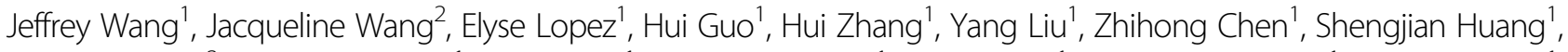 \\ Shouhao Zhou', Angela Leeming ${ }^{3}$, R. J. Zhang ${ }^{1}$, Dayoung Jung (1)', Hannah Shi', Hadley Grundman', Darian Doakes ${ }^{1}$, \\ Kathleen Cui ${ }^{1}$, Changying Jiang ${ }^{1}$, Makhdum Ahmed ${ }^{1}$, Krystle Nomie', Bingliang Fang ${ }^{2}$, Michael Wang $\mathbb{B}^{1,4}$, \\ Yixin Yao (1) and Liang Zhang'
}

Dear Editor,

B-cell lymphomas such as relapsed or refractory mantle cell lymphoma (MCL) and diffuse large B-cell lymphoma (DLBCL) are aggressive non-Hodgkin lymphomas (NHL). Although the BTK inhibitor ibrutinib has offered markedly improved clinical outcomes after disease relapse from multiple prior therapies ${ }^{1,2}$, ibrutinib resistance often develops in MCL, even following initial positive responses, and DLBCL is frequently resistant to ibrutinib ${ }^{2,3}$. We and others have found that specific tumor suppressor gene defects are correlated with relapsed/refractory characteristics and cause poor clinical outcomes ${ }^{4,5}$. However, these specific tumor suppressors, including TP53 and CDKN2A, are not druggable, and indirectly targeting these tumor suppressor-mediated pathways has not resulted in high clinical response rates. Auranofin, a gold-containing compound that is FDA-approved for treatment of rheumatoid arthritis, is being repurposed as a potential anti-tumor drug against different refractory malignancies ${ }^{6}$. Currently, auranofin is being assessed in clinical trials for chronic lymphocytic leukemia, breast cancer, and lung cancer (NCT01747798, NCT01419691, and NCT01737502, respectively). Auranofin has a proven safety profile, making it an attractive compound for clinical trials ${ }^{7}$, as an estimated $70-90 \%$ of agents fail clinical trials, with safety concerns being a major cause of drug development discontinuation ${ }^{8}$. However, repurposing FDA-approved drugs with known safety in humans for

\footnotetext{
Correspondence: Liang Zhang (liazhang@mdanderson.org)

${ }^{1}$ Department of Lymphoma and Myeloma, The University of Texas MD

Anderson Cancer Center, Houston, TX, USA

${ }^{2}$ Department of Thoracic Surgery, The University of Texas MD Anderson Cancer Center, Houston, TX, USA

Full list of author information is available at the end of the article.

These authors contributed equally: Jeffrey Wang, Jacqueline Wang, Elyse Lopez
}

new indications may circumvent these issues and offer a promising new area of investigation. Here, we demonstrate that auranofin targets thioredoxin reductase-1 (Txnrd1) to effectively induce DNA damage, reactive oxygen species (ROS) production, cell growth inhibition, and apoptosis in aggressive B-cell lymphomas, especially in TP53-mutated or PTEN-deleted lymphomas. First, auranofin has consistently shown to induce lethality in a panel of DLBCL and MCL cell lines. We treated 8 DLBCL cell lines-including three GCB-type cell lines (OCI-Ly8, OCI-Ly7, and Su-DHL-10) and five ABC-type cell lines (OCL-Ly3, OCI-Ly10, U2932, TMD8, and HBL-1) -and six MCL cell lines (Z-138, JVM-2, Mino, Maver-1, Jeko-1, and Jeko-R) with auranofin in concentrations ranging from 0 to $5 \mu \mathrm{M}$ for $72 \mathrm{~h}$ and tested cell viability using a luminescent assay. We found that auranofin was cytotoxic to both DLBCL (Fig. 1a) and MCL (Fig. 1b) in a dosedependent manner, with an $\mathrm{IC}_{50}$ range of 0.058-1.389 $\mu \mathrm{M}$. TP53-mutated lymphoma cells, Mino, Maver-1, OCI-Ly7, OCI-Ly8, Su-DHL-10, U2932, JeKo-1, and JeKo-R, as well as PTEN-lost Z-138 were much more sensitive to auranofin. However, the TP53-intact lymphoma cell lines OCI-Ly3 and JVM-2 had much higher $\mathrm{IC}_{50}$ values than the other cell lines (Fig. 1c). The JeKo- $\mathrm{R}$ cell line consists of ibrutinib-resistant MCL cells established by long-term exposure to an escalating dose of ibrutinib during JeKo-1 cell culture, and it represents acquired resistance to ibrutinib. Z-138 has intrinsic resistance to ibrutinib due to its constitutive NIK signaling activation ${ }^{9}$. Our data demonstrate that the $\mathrm{IC}_{50}$ of auranofin in Z-138 is only $0.058 \mu \mathrm{M}$, which is 10 -fold lower than the $\mathrm{IC}_{50}$ of the PTEN- or TP53-intact lymphoma cell lines (Fig. 1c). 


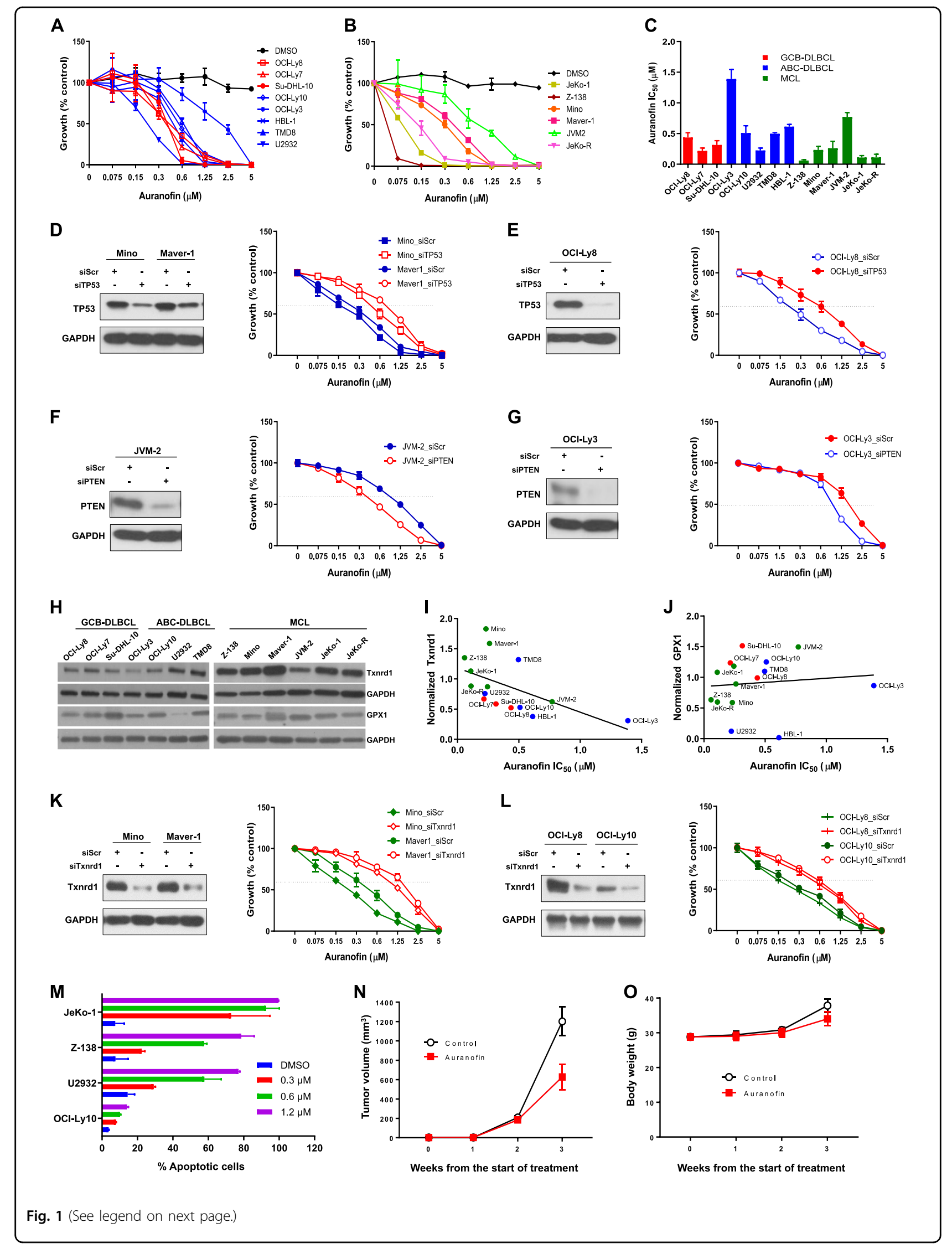


(see figure on previous page)

Fig. 1 Auranofin-induced cytotoxicity to lymphoma cells is related to Txnrd1 but not GPX1. a Dose-response curve for DLBCL treated with auranofin for $72 \mathrm{~h}$. Red = GCB type; Blue = ABC type. $\mathbf{b}$ Dose-response curve for various MCL cell lines treated with auranofin for $72 \mathrm{~h}$. $\mathbf{c}$ Averaged auranofin $I_{50}$ calculated from repeated non-linear regression of dose-response curves. $\mathbf{d}$ Mino, Maver-1, and e OCl-Ly8 cell lines were

immunoblotted by anti-TP53 antibody after 48-h knockdown of TP53 by siRNA (siTP53) and scrambled siRNA (siScr), and auranofin-treated doseresponse growth curves were shown at the presentence of siTP53 or siScr for $72 \mathrm{~h}$. $\mathbf{f} \mathrm{JVM}-2$ and $\mathbf{g}$ OCl-Ly3 cell lines were immunoblotted by antiPTEN antibody after 48-h knockdown of PTEN by siRNA (siPTEN) and siScr, and auranofin-treated dose-response growth curves were shown at the presentence of siPTEN or siScr for $72 \mathbf{h}$. $\mathbf{h}$ Immunoblot of Txnrd1 and GPX1 in MCL and DLBCL. $\mathbf{i}$ Txnrd1 protein level, and $\mathbf{j}$ GPX1 protein level to the relation with $I_{50}$ of auranofin in $\mathrm{DLBCL}$ and $\mathrm{MCL}$ cell lines. A Pearson's correlation test was performed with $p<0.05$ being treated as significant. k Mino, Maver-1, and I. OCI-Ly8 and OCI-Ly10 cell lines were immunoblotted by anti-Txnrd1 antibody after 48-h knockdown of Txnrd1 by siRNA (siTxnrd1) and siScr, and auranofin-treated dose-response growth curves were shown at the presentence of siTP53 or siScr for $72 \mathrm{~h}$. $\mathbf{m}$ Auranofininduced apoptosis at $24 \mathrm{~h}$ as measured by Annexin-V binding assay. $\mathbf{n}$ The in vivo effects of auranofin in TP53-mutated DLBCL PDX model. Mice were administered vehicle control or auranofin $50 \mathrm{mg} / \mathrm{kg}$, oral gavage, daily for 21 consecutive days after 3 days of tumor engraftment. Tumor burden was calculated by measuring tumor volume $(n=5$; auranofin vs. vehicle, $p=0.000213)$. o Body weight was calculated during drug treatment (auranofin vs. vehicle, $p=0.01556$ ).

To address the correlation of auranofin and TP53 mutation and PTEN loss, in TP53-mutated MCL cell lines Mino, Maver-1 (Fig. 1d), and DLBCL cell line OCI-Ly8 (Fig. 1e), TP53 knockdown made cells more tolerant to auranofin treatment (each pair cell lines siTP53 vs siScr, $p<0.0001)$. However, TP53 knockdown in two TP53 wild-type cell lines JVM-2 and OCI-Ly3 did not affect the changes of growth inhibition after auranofin treatment (data not shown), indicating that only mutated-TP53 sensitized cells to auranofin. Next, we specifically knocked down PTEN in JVM-2 (Fig. 1f) and OCI-Ly3 (Fig. 1g), cells became more sensitive to auranofin treatment (each pair cell lines siPTEN vs siScr, $p<0.0001$ ). The result is consistent with the data in PTEN-lost Z-138 cells (Fig. 1c) indicating PTEN loss sensitized cells to auranofin treatment.

A recent publication shows that the main function of auranofin is to inhibit Txnrd1 in the cytoplasm and nucleus to induce ROS production, tumor cell growth inhibition, and apoptosis ${ }^{10}$. Txnrd1 could therefore be the therapeutic target of auranofin for lymphoma. In addition, TP53 and PTEN regulate glutathione perosidase-1 (GPX1), which results in ROS accumulation and cell damage ${ }^{11}$. Therefore, we investigated the expression of both Txnrd1 and GPX1 in lymphoma cells, especially TP53-mutated or PTEN-deleted lymphoma cells, and explored their correlation with auranofin treatment. We found that all 14 tested aggressive lymphoma cell lines expressed Txnrd1 and GPX1, but the TP53-mutated cell line U2932 had very low GPX1 protein levels (Fig. 1h). We used a Pearson's correlation to evaluate the correlation between Txnrd1 or GPX1 protein levels with the auranofin $\mathrm{IC}_{50}$. We found a significant inverse correlation between Txnrd 1 and the auranofin $\mathrm{IC}_{50}$ (Fig. $1 \mathrm{i}, p=$ 0.036). However, GPX1 protein levels did not correlate with auranofin $\mathrm{IC}_{50}$ (Fig. $1 \mathrm{j}, p=0.7168$ ). The results indicate that auranofin-induced cytotoxicity to lymphoma cells is related to Txnrd1 protein levels but not to GPX1. To further address the correlation of auranofin to Txnrd1,
Txnrd1 knockdown in Mino, Maver-1 (Fig. 1k), and OCILy8 and OCI-Ly10 (Fig. 11) caused cells more tolerant to auranofin treatment (each pair cell lines siTxnrd1 vs siScr, $p<0.0001$ ).

Next, to investigate how auranofin induces apoptosis, we treated the TP53 or PTEN wild-type/mutated/deleted cell lines with $0-1.2 \mu \mathrm{M}$ auranofin for $24 \mathrm{~h}$, and a dosedependent apoptosis was observed in all of these cell lines. We observed that the TP53-mutated cell lines U2932 and JeKo-1 and the PTEN-lost cell line Z-138, were much more sensitive to auranofin treatment than the TP53 and PTEN wild-type cell line OCI-Ly10 (Fig. 1m). To validate the therapeutic effect of auranofin in vivo, we established a DLBCL patient-derived xenograft (PDX) model using freshly isolated tumor cells from a TP53-mutated DLBCL patient sample. We found that auranofin significantly inhibited tumor growth during 21 consecutive days of $50 \mathrm{mg} / \mathrm{kg}$ auranofin treatment by oral gavage (Fig. $1 \mathrm{n}, p=$ $0.000213)$. All mice were in good body condition during treatment, and there was no body weight difference between the vehicle and auranofin treatment cohorts (Fig. 1o, $p=0.1556)$.

To elucidate the mechanism of action of auranofin in lymphoma cells, we first showed that auranofin completely inactivated Txnrd1 activity in vitro (Fig. 2a). Twohour pretreatment with $5 \mathrm{mM}$ antioxidant $\mathrm{N}$ acetylcysteine (NAC), which is a ROS inhibitor, significantly reduced the production of ROS as shown by a significant increase of H2DCFDA fluorescence (Fig. 2b, c, all $p<0.0001)$. To probe the mechanism of auranofininduced cell death, cells were then treated with auranofin and co-incubated with either $5 \mathrm{mM} \mathrm{NAC}$ or $20 \mu \mathrm{M} \mathrm{Z}$ VAD-FMK, a pan-caspase inhibitor. Incubation with either NAC or Z-VAD-FMK abrogated auranofin-induced apoptosis (Fig. $2 \mathrm{~d}, p<0.05$ ). The results demonstrate that auranofin induced ROS- and caspase-dependent apoptosis. Furthermore, treatment with $1.2 \mu \mathrm{M}$ or $2.5 \mu \mathrm{M}$ auranofin reduced the mitochondrial membrane potential at $12 \mathrm{~h}$ (Fig. 2e, $p<0.05$ ). Since long-term exposure to high 


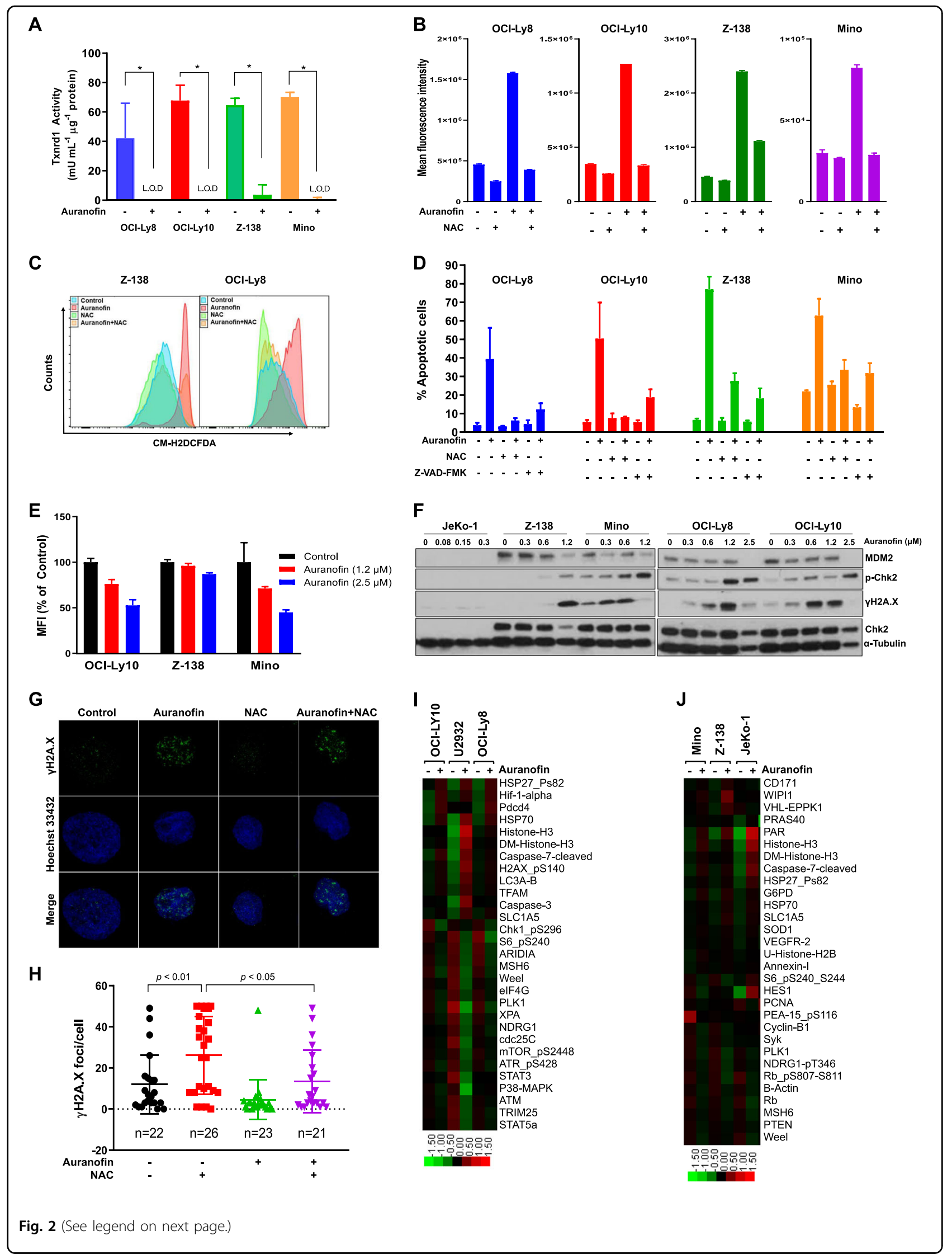


(see figure on previous page)

Fig. 2 Auranofin inhibits Txnrd1, induces ROS accumulation, DNA damage, cell growth inhibition, and ROS- and caspase-dependent apoptosis in B-cell lymphomas. a Lysates from cells treated with $1.2 \mu \mathrm{M}$ auranofin for $24 \mathrm{~h}$ were evaluated for Txnrd 1 activity (auranofin vs. vehicle control, two-sample $t$-test, all $p<0.05$ ). b Pool data and $\mathbf{c}$ Representative data showed that auranofin significantly increased H2DCFDA fluorescence (two-sample $t$-test, all $p<0.0001$ ). Cells were treated with $5 \mu \mathrm{M}$ auranofin with/without $5 \mathrm{mM} \mathrm{NAC}$ for $2 \mathrm{~h}$, and fluorescence was detected by flow cytometry. d Cell lines OCI-Ly8, OCI-Ly10, Z-138, and Mino were treated with $1.2 \mu \mathrm{M}$ auranofin in the presence of either 5 mM NAC or $20 \mu \mathrm{M}$ Z-VADFMK for $24 \mathrm{~h}$. Apoptosis was measured by Annexin-V binding assay. e Cells were treated with the indicated doses of auranofin for $12 \mathrm{~h}$ and subjected to mitochondrial membrane potential assay (linear regression, all $p<0.05$ ). f Immunoblot of JeKo-1, Z-138, Mino, OCl-Ly8, and OCl-Ly10 after 24-h auranofin treatment showing DNA damage response. $\mathbf{g}$ Representative super resolution maximum intensity projections of whole cell micrographs of Z-138 cells treated with $1.2 \mu \mathrm{M}$ auranofin with/without $5 \mathrm{mM} \mathrm{NAC}$ for $24 \mathrm{~h}$. Immunofluorescence staining for $\gamma \mathrm{H} 2 \mathrm{~A} . \mathrm{X}$ and Hoechst is shown. $\mathbf{h}$ $\gamma \mathrm{H} 2 \mathrm{~A}$.X foci were quantitated using the FindFoci ImageJ plugin. At least randomly 20 randomized cells of each treatment condition were evaluated (auranofin vs. vehicle control, $p<0.01$ ). NAC prevented auranofin-induced DNA damage (auranofin vs. auranofin plus NAC, $p<0.05$ ). i DLBCL cell lines OCI-Ly10, U2932, and OCI-Ly8, and j MCL cell lines Mino, Z-138, and JeKo-1 were treated with $1.2 \mu \mathrm{M}$ auranofin for $24 \mathrm{~h}$ and then subjected to RPPA analysis. The top 30 most differentially expressed proteins are displayed.

levels of ROS causes DNA damage, we then investigated the effects of auranofin on DNA damage response pathways. We found that auranofin dose-dependently activated CHK2 and $\gamma \mathrm{H} 2 \mathrm{~A} . \mathrm{X}$, as well as an observed concomitant decrease of MDM2 in Z-138, Mino, OCILy8, and OCI-Ly10, suggesting TP53 release and activation in these TP53-intact lymphoma cell lines (Fig. 2f). Next, immunofluorescent staining with anti- $\gamma \mathrm{H} 2 \mathrm{~A} . \mathrm{X}$ confirmed that auranofin-treated Z-138 cells had significantly more phosphorylated H2A.X than control cells (Fig. 2g, $p=0.0118$ ) and that $5 \mathrm{mM}$ NAC generally inhibited H2A.X phosphorylation (Fig. 2h, $p=0.0211$ ). These results demonstrate that these TP53-intact lymphoma Z-138 cells are DNA damaged during ROSdependent apoptosis induced by auranofin treatment, regardless of PTEN loss. Interestingly, TP53-mutated JeKo-1 cells had no DNA damage response (Fig. 2f), indicating that auranofin-induced cytotoxicity in TP53mutated JeKo-1 cells is not dependent on the DNA damage pathway activation.

To further investigate other pathways that auranofin targets in both TP53 wild-type and mutated lymphoma cells, we performed RPPA analysis on OCI-Ly8, OCILy10, Mino, Z-138, U2932, and JeKo-1 cell lines. The top 30 most differentially expressed proteins were analyzed in two independent RPPA data analyses of DLBCL (Fig. 2i) and MCL (Fig. 2j). We found that TP53-mutated DLBCL and MCL cells have conspicuous changes of protein expression, which were totally different from TP53 wildtype cells. Especially, in TP53-mutated DLBCL cell line U2932, auranofin increased the expression levels of HSP70, histone H3, caspase-3, 7, p-H2A.X, LC3A, and SLC1A5, and decreased the expression of p-S6, ARID1A, MSH6, Wee1, eIF4G, PLK1, XPA, p-NDRG1, Cdc25C, mTOR, ATR, STAT3, ATM, and TRIM25 (Fig. 2i). In TP53-mutated MCL cell line JeKo-1, auranofin increased the expression levels of PAR, histone H3, caspase-7, HSP27, and SLC1A5, and decreased the expression of HES1, p-Rb, and Weel (Fig. 2j). The common pattern is that auranofin increased the H3 and SLC1A5 levels and decreased Wee1 expression in both TP53-mutated DLBCL cell line U2932 and MCL cell line JeKo-1. SLC1A5 is a glutamine transporter ${ }^{12}$, and Wee1 regulates DNA damage checkpoints ${ }^{13}$. Auranofin may strongly induce metabolic stress, as evidenced by reducing the mitochondrial membrane potential and then increasing the expression of SLC1A5 as compensation for more nutrient supplements from glutaminolysis. In addition, auranofin increases the pro-autophagic protein LC3A and decreases the proteins for signaling activation of mTOR, STAT3, and the cell cycle in TP53-mutated DLBCL cell line U2932, indicating that auranofin may have more mechanisms for treating TP53-mutated DLBCL.

In summary, our study demonstrates that auranofin exerts its anti-lymphoma cytotoxic effects through ROSbased therapeutics by targeting Txnrd1. Auranofin induces DNA damage, cell growth inhibition, and ROS- and caspase-dependent apoptosis in aggressive B-cell lymphomas, and it especially shows more significant therapeutic effects on TP53-mutated or PTEN-deleted lymphomas. Our brief study points out that auranofin may be repurposed as an effective clinical option for TP53-mutated or PTEN-deleted refractory B-cell lymphoma.

\begin{abstract}
Acknowledgements
Cell line authentication was performed by the MD Anderson Cancer Center Characterized Cell Line Core Facility, funded by grant NCI \#CA016672.

Microscopy data were collected at the MD Anderson Cancer Center advanced microscopy core facility. Microscopic data reported in this publication was supported by the National Institutes of Health under award number NIH \#1S10 RR029552. Reverse phase protein array data were performed at the MD Anderson RPPA core facility. The RPPA data reported were supported by the National Cancer Institute under award number NCI \#CA16672. The content is solely the responsibility of the authors and does not necessarily represent the official views of the National Institutes of Health. We thank Ms. Kelley P. Murfin for editing this manuscript.
\end{abstract}

\section{Funding}

Philanthropic funds from The Gary Rogers Foundation and the Kinder Foundation. 


\section{Author details}

'Department of Lymphoma and Myeloma, The University of Texas MD Anderson Cancer Center, Houston, TX, USA. '2Department of Thoracic Surgery, The University of Texas MD Anderson Cancer Center, Houston, TX, USA. ${ }^{3}$ Department of Biostatistics, The University of Texas MD Anderson Cancer Center, Houston, TX, USA. ${ }^{4}$ Department of Stem Cell Transplantation and Cellular Therapy, The University of Texas MD Anderson Cancer Center, Houston, TX, USA

\section{Author contributions}

M.W., B.F. and L.Z. designed the study. J.W., J.W. and E.L. initiated the project and performed the experiments. H.G., H.Z., Y.L., Z.C., S.H., A.L., R.Z., C.J., M.A. K.N. and Y.Y. contributed to the project by providing key experimental techniques and ideas. D.J., H.S., H.G., D.D. and K.C. provided analytic and laboratory support. J.W. wrote the draft, and M.W., B.F., K.N., Y.Y. and L.Z. wrote and revised the manuscript. S.Z. performed the statistical analysis.

\section{Conflict of interest}

M.W. receives research funding from Janssen, AstraZeneca, Acerta Pharmaceuticals, Kite Pharmaceuticals, Juno Therapeutics, BeiGene, Novartis, Celgene, Biolnvent, Oncternal Therapeutics, Loxo Oncology, VelosBio, and Karus Therapeutics, but does not have competing interests related to this work. All other authors declare no conflicts of interest.

\section{Publisher's note}

Springer Nature remains neutral with regard to jurisdictional claims in published maps and institutional affiliations.

Supplementary Information accompanies this paper at (https://doi.org/ 10.1038/s41408-019-0259-8).

Received: 6 June 2019 Revised: 6 November 2019 Accepted: 13 November 2019

Published online: 28 November 2019

\section{References}

1. Wang, M. L. et al. Targeting BTK with ibrutinib in relapsed or refractory mantlecell lymphoma. N. Engl. J. Med. 369, 507-516 (2013).

2. Wilson, W. H. et al. Targeting B cell receptor signaling with ibrutinib in diffuse large B cell lymphoma. Nat. Med. 21, 922-926 (2015).

3. Cheah, C. Y. et al. Patients with mantle cell lymphoma failing ibrutinib are unlikely to respond to salvage chemotherapy and have poor outcomes. Ann. Oncol. 26, 1175-1179 (2015).

4. Karube, K. et al. Integrating genomic alterations in diffuse large B-cell lymphoma identifies new relevant pathways and potential therapeutic targets. Leukemia 32, 675-684 (2018).

5. Eskelund, C. W. et al. TP53 mutations identify younger mantle cell lymphoma patients who do not benefit from intensive chemoimmunotherapy. Blood 130, 1903-1910 (2017).

6. Fiskus, W. et al. Auranofin induces lethal oxidative and endoplasmic reticulum stress and exerts potent preclinical activity against chronic lymphocytic leukemia. Cancer Res. 74, 2520-2532 (2014).

7. Heuer, M. A., Pietrusko, R. G., Morris, R. W. \& Scheffler, B. J. An analysis of worldwide safety experience with auranofin. J. Rheumatol. 12, 695-699 (1985).

8. Woodcock, J. \& Woosley, R. The FDA critical path initiative and its influence on new drug development. Ann. Rev. Med. 59, 1-12 (2008).

9. Rahal, R. et al. Pharmacological and genomic profiling identifies NF-kappaBtargeted treatment strategies for mantle cell lymphoma. Nat. Med. 20, 87-92 (2014).

10. Lee, D. et al. Induction of oxidative stress via inhibition of thioredoxin reductase 1 is an effective therapeutic approach for hepatocellular carcinoma. Hepatology 69, 1768-1786 (2018).

11. $\mathrm{Wu}, \mathrm{Y}$. et al. PTEN phosphorylation and nuclear export mediate free fatty acidinduced oxidative stress. Antioxid Redox Signal 20, 1382-1395 (2014).

12. van Geldermalsen, M. et al. ASCT2/SLC1A5 controls glutamine uptake and tumour growth in triple-negative basal-like breast cancer. Oncogene 35, 3201-3208 (2016).

13. Geenen, J. J. J. \& Schellens, J. H. M. Molecular pathways: targeting the protein kinase Wee1 in cancer. Clin. Cancer Res. 23, 4540-4544 (2017). 